\title{
Extent of Emphysema on HRCT Affects Loss of Fat-free Mass and Fat Mass in COPD
}

\author{
Hiroko Kurosaki ${ }^{1}$, Takeo Ishii ${ }^{1}$, Norihisa Motohashi ${ }^{1}$, Takashi Motegi ${ }^{1}$, Kouichi Yamada ${ }^{1}$, \\ Shoji Kudoh ${ }^{1}$, Rupert C.M. Jones ${ }^{2}$ and Kozui Kida ${ }^{1}$
}

\begin{abstract}
Background Although muscle loss is thought to be a prognostic factor in chronic obstructive pulmonary disease (COPD), its determinants remain unclear.

Aim To verify the hypothesis that fat-free mass (FFM) and fat mass (FM) are associated with the extent of emphysema in COPD patients.

Patients and Methods A total of 112 stable, male current or ex-smokers with or without COPD attending a secondary care specialist COPD clinic were studied. FFM and FM were measured by bioelectrical impedance analysis. We also assessed the nutrition status, muscle strength by the handgrip test, exercise tolerance by the 6-minute walking test, airflow limitation and diffusion capacity, the extent of emphysema by highresolution CT scan, systemic inflammation status using C-reactive protein, and a lipid-related hormone (adiponectin).

Results The FFM index (FFMI), which was defined as the FFM divided by the square of the body height, was significantly correlated with age, the total number of lymphocytes, handgrip strength, distance on 6minute walking, airflow limitation, diffusion capacity, extent of emphysema, and C-reactive protein. On multivariate analysis, the FFMI was associated with handgrip strength and inversely correlated with the extent of emphysema. The FM index (FMI) was positively correlated with pack-years, and was inversely correlated with the extent of emphysema and concentrations of adiponectin.
\end{abstract}

Conclusion The extent of emphysema was correlated with skeletal muscle loss and also the FM.

Key words: chronic obstructive pulmonary disease, fat-free mass, fat mass, low attenuation area, muscle strength

(Inter Med 48: 41-48, 2009)

(DOI: 10.2169/internalmedicine.48.1102)

\section{Introduction}

Chronic obstructive pulmonary disease (COPD) is characterized by not only airflow limitation but also some extrapulmonary comorbidities (1). In particular, skeletal muscle weakness and atrophy are frequently observed in patients with COPD (2); skeletal muscle weakness is associated with a reduced fat-free mass (FFM) of the extremities but not with airflow limitation (3).

The pathogenesis of muscle wasting in patients with COPD has attracted considerable research interest. A reduc- tion of physical activity may be responsible for deconditioning, in which metabolic causes have been examined in detail (4). Anabolic/catabolic disturbance in COPD leads to a shift toward catabolism, which possibly induces the development of peripheral muscle-wasting (5); selective type-IIX and IIA/ IIX atrophy accompanies an increase in fibrosis and fat cell replacement $(6,7)$, and skeletal muscle apoptosis increases in COPD (8). However, it is not known whether these changes are specific to COPD, since similar muscle-wasting is associated with inflammatory changes in patients with chronic diseases such as those on long-term hemodialysis (9) or with chronic heart disease (10). The relationship be-

${ }^{1}$ Department of Pulmonary Medicine, Infection, and Oncology, Respiratory Care Clinic, Nippon Medical School, Tokyo and ${ }^{2}$ Respiratory Research Unit, Peninsula Medical School, Plymouth, UK

Received for publication March 11, 2008; Accepted for publication September 4, 2008

Correspondence to Dr. Kozui Kida, kkida@nms.ac.jp 
tween skeletal muscle dysfunction, aging and immobility is not clear $(11,12)$. Immobilization in advanced aging (13), severe dyspnea in COPD (14) or combined with chronic disease and immobilization (15) may result in muscle-wasting. This muscle-wasting is associated with a poor prognosis, and if FFM depletion can be reversed, the prognosis also improves (16).

Despite the fact that the fat mass (FM) cannot be used to predict COPD survival, FM could be associated with comorbidities in COPD patients, since a recent review (17) showed that COPD has extra-pulmonary effects, including an increased risk of cardiovascular disease, osteoporosis, and several neurologic defects. FM is reported to be an independent risk factor for myocardial infarction (18) and to be associated with systemic inflammation (19). All these reports suggest that FM plays a critical role in the pathogenesis of systemic effects in COPD.

In this study, we hypothesized that FFM and FM are associated with the extent of emphysema in COPD patients. We comprehensively investigated the determinants of the fat-free mass and fat mass, especially in relation to: (i) lung structure and function, (ii) exercise tolerance, and (iii) markers of systemic inflammation and lipid metabolism.

\section{Materials and Methods}

\section{Parameters}

We investigated the correlations between FFM or FM and the following parameters:

a. Nutrition status (total protein, total number of lymphocytes)

b. Muscle strength (handgrip strength)

c. Exercise tolerance (6-minute walking test (6 MWT))

d. Severity of COPD: air-flow limitation (forced expiratory volume in 1 second (FEV1)), the extent of emphysema on high-resolution computed tomography (HRCT), and the measured percentage of the low-attenuation area (20)

e. Systemic inflammation (highly-sensitive C-reactive protein: CRP)

f. Hormones related to lipid metabolism (adiponectin), since it is known that adiponectin is closely correlated to muscle mass (21)

\section{Subjects}

The subjects were consecutive male patients who initially consulted the outpatient clinic of the Respiratory Care Clinic, Nippon Medical School, Tokyo, Japan, a secondary care specialist COPD clinic, for ambulatory treatment in a stable condition from October 2005 to December 2005. We only studied male subjects in this study since there is so much difference between males and females on FFMI or FMI and there are much more male than female COPD patients, especially in Japan (22). Eligible patients fulfilled the following criteria: 1) patients whose clinical course, clinical symptoms, and laboratory data satisfied criteria for the clini- cal diagnosis of COPD according to the COPD guideline published by the American Thoracic Society and European Respiratory Society (23), and smokers without COPD who have cough, sputum or dyspnea, and 2) those with a history of smoking, including current and ex-smokers (number of pack-years $>20$ ).

We excluded patients with the following:

1) with a history of atopy or any apparent clinical features of asthma,

2) those receiving any systemic corticosteroid regimen,

3) with exacerbations during the preceding three months,

4) with other respiratory diseases such as bronchiectasis or any pulmonary fibrosis, and

5) with those cardiac disorders, such as congestive heart failure.

This study was approved by the ethics committee of the institute, and the subjects were enrolled after appropriate informed consent was obtained.

\section{Anthropometry}

Body height was determined to the nearest $0.1 \mathrm{~cm}$ with subjects standing barefoot (BWB-800; Tanita Co., Tokyo, Japan). Body weight was measured without shoes and with light clothing to the nearest $0.1 \mathrm{~kg}$ (Rainbow; Tanita; Tokyo, Japan). The body mass index (BMI) was calculated as the ratio of weight to height in meters squared. FFM and FM were measured by bioelectrical impedance analysis at frequencies of 5, 50, 250, and $500 \mathrm{kHz}$ (Inbody 3.2 ; Biospace Japan Inc., Tokyo, Japan), and the FFM index (FFMI) and FM index (FMI) were calculated as the ratio of FFM or FM, respectively, to the height in meters squared.

\section{Assessment of nutrition status, inflammation, and a lipid-related hormone}

We measured the concentration of total protein and lymphocytes, which are thought to reflect the nutrition status, highly-sensitive CRP (BN-II; Dade Behring Co., Tokyo, Japan), which is closely correlated to the severity of COPD (24), and serum adiponectin (Microplate EIA device Plato 3300; Otsuka Pharmaceutical Co., Tokyo, Japan), which is closely associated with lipid metabolism (21).

\section{Muscle strength}

Handgrip strength was measured with a hand dynamometer (GRIP-A; Takei Scientific Instruments Co., Niigata, Japan). Three measurements were made using each hand with the arm unsupported, and the maximal grip strength for each hand was used.

\section{Exercise capacity}

The 6 MWT was conducted according to ATS standards (25). During the 6 MWT, oximetry was performed every 10 seconds with a pulse oximeter (Pulseox; Teijin Co., Tokyo Japan). 


\section{Pulmonary function testing}

Pulmonary function parameters, including postbronchodilator FEV1, carbon monoxide diffusing capacity (DLCO), vital capacity (VC), and forced vital capacity (FVC), were measured according to the American Thoracic Society guidelines (26) using equipment for lung function testing with computer processing (Chestac; Chest Co., Tokyo, Japan). The reference values of post-bronchodilator FEV1 and VC were used according to the standards of the Japanese Respiratory Society (27).

\section{Quantitative analysis on CT}

We calculated the extent of the low-attenuation area (\% LAA) by HRCT scan, according to Nakano et al, with a slight modification (20). Briefly, six slices were obtained from the bilateral lungs at upper, middle, and lower lobes. The upper slice was obtained at the aortic arch, the middle section was taken at the carina, and the lower section was taken approximately 2-3 cm above the top of the diaphragm. HRCT was performed using $1.25-\mathrm{mm}$ collimation, a scan time (rotation time) of 0.8 seconds, $120 \mathrm{kV}$, and 100-600 mA with a Light Speed Pro16 CT scanner (GE Co., Tokyo, Japan). The \%LAA was calculated by the scanner on these 6 slices. The arithmetical mean value for each case was used as a representative value as \%LAA. The cutoff level between the normal lung density and LAA was defined as $940 \mathrm{HU}$.

\section{Statistical analysis}

The results are presented as the mean (standard deviation) for all values. Pearson's correlation analyses were used to establish variables correlated with FFMI or FMI. Backwards stepwise multiple linear regression was employed to select the variables that affect FFMI or FMI in multivariate analyses, with the parameters significantly related by Pearson's correlation analyses. Data were analyzed using the Statistical Package for the Social Sciences (SPSS), Version 11.0.1, for Windows (SPSS Inc., Chicago, IL, U.S.A.). A p-value of < 0.05 was considered significant.

\section{Results}

\section{Characteristics of the subjects}

A total of 112 male smokers (27 current and 85 exsmokers) with or without COPD classified according to clinical guidelines (23) were included in this study: smokers without COPD ( $n=44)$, I ( $n=7)$, II $(n=26)$, III $(n=26)$, and IV $(n=9)$. The characteristics of the subjects are shown in Table 1 .

\section{Determinants of FFM in COPD}

We first performed Pearson's correlation analyses between FFMI and various clinical parameters or some basic characteristics (age and pack-years) to detect simple correlations
(Table 2). FFMI was significantly correlated with the following clinical parameters: age, total number of lymphocytes, handgrip strength, distance on 6 MWT, FEV1\% predicted, DLCO, \%LAA, and highly-sensitive CRP. Then, we conducted multivariate analysis for FFMI with these clinical parameters except for CRP and adiponectin, first including all subjects $(n=112)$, and then we performed the same analyses for subjects in whom which we had data on both the serum concentration of CRP $(n=75)$ and adiponectin $(n=34)$. Since there were close correlations between \%LAA and FEV1\% predicted $(\mathrm{r}=-0.605, \mathrm{p}<0.001)$ or DLCO $(\mathrm{r}=-0.524$, $\mathrm{p}<0.001$ ), we had to select one factor out of three to perform this multivariate analysis. As it was associated with FFM and also with FM (as written below) after Pearson's correlation analyses, \%LAA could be a useful predictor for body composition of COPD patients in a comprehensive manner, which leads us to select \%LAA instead of \%FEV1 and DLCO for a further multivariate analysis against FFMI. In the results, in the multivariate analysis for all subjects, FFMI was associated with handgrip strength and inversely correlated with \%LAA (Table 3 and Fig. 1A), and these correlations remained with significance after adjustment with age (data not shown). FFMI was not associated with highlysensitive CRP on multivariate analysis. However, \%LAA was not significantly correlated with FFMI by univariate analysis $(p=0.09)$ when smokers without COPD were excluded.

\section{Determinants of FM in COPD}

FMI was also inversely correlated with \% LAA and associated with both pack-years and adiponectin on univariate analysis (Table 2). Although FFMI was significantly associated with \%FEV1 as described above, FMI was not. The correlation between FMI and \%LAA or pack-years remained after multivariate analysis involving all subjects (Table 4 and Fig. 1B), or with adjustment of age (data not shown).\%LAA was significantly correlated with FMI by univariate analysis ( $p=0.001)$ when smokers without COPD were excluded.

\section{Discussion}

This study revealed several interest findings.

First, the extent of emphysema on HRCT was significantly correlated with both the FFM and FM. Further, we showed that muscle strength in COPD was correlated with muscle wasting. It should be noted in the present study that FFMI was associated with the severity of emphysema (\% LAA) on HRCT, which is consistent with a previous report (3); however, our study design differed and appeared more precise; the severity of emphysema was assessed qualitatively previously (3), whereas the percentage of emphysema was strictly measured in the present study. Our data also indicated that DLCO and FEV1\% predicted were correlated with FFMI, and the correlation coefficient was similar to that of \%LAA (Table 2). Thus, there is a possibility that DLCO or FEV1\% predicted would be useful values to pre- 
Table 1. Characteristics of the Subjects

\begin{tabular}{|c|c|c|}
\hline Subjects (n) & \multicolumn{2}{|l|}{$\mathrm{n}=112$} \\
\hline Age (yrs) & 69.2 & $(10.4)$ \\
\hline $\operatorname{BMI}\left(\mathrm{kg} / \mathrm{m}^{2}\right)$ & 22.5 & $(3.2)$ \\
\hline FFMI $\left(\mathrm{kg} / \mathrm{m}^{2}\right)$ & 17.2 & (1.9) \\
\hline FMI $\left(\mathrm{kg} / \mathrm{m}^{2}\right)$ & 5.3 & $(1.8)$ \\
\hline Pack-years & 65.4 & $(39.5)$ \\
\hline Stages by ATS/ERS & \multicolumn{2}{|c|}{ (smokers without COPD: 44, I: 7,II:26, III: 26, IV: 9) } \\
\hline FEV1 (L) & 1.94 & $(8.48)$ \\
\hline FEV1 \% (\%) & 62.9 & $(15.9)$ \\
\hline FEV1 $\%$ predicted $(\%)$ & 64.5 & $(25.2)$ \\
\hline $\mathrm{VC}(\mathrm{L})$ & 3.23 & $(0.84)$ \\
\hline$\% \mathrm{VC}(\%)$ & 89.8 & $(17.7)$ \\
\hline $\mathrm{DLCO}(\mathrm{mL} / \mathrm{m} / \mathrm{mmHg})$ & 12.8 & $(5.5)$ \\
\hline \multicolumn{3}{|l|}{ HRCT } \\
\hline$\%$ LAA mean $(\%)$ & 28.7 & $(15.6)$ \\
\hline \multicolumn{3}{|l|}{ 6MWT } \\
\hline distance $(m)$ & 464 & (119) \\
\hline Handgrip strength $(\mathrm{kg})$ & 33.3 & (7.9) \\
\hline \multicolumn{3}{|l|}{ Serum markers } \\
\hline Adiponectin $(\mu \mathrm{g} / \mathrm{mL})$ & 11.8 & $(6.9)$ \\
\hline $\mathrm{CRP}(\mathrm{mg} / \mathrm{dL})$ & 0.14 & $(0.24)$ \\
\hline Total protein $(\mathrm{g} / \mathrm{dL})$ & 7.12 & $(0.43)$ \\
\hline \multicolumn{3}{|c|}{ Total number of lymphocytes } \\
\hline$(/ \mu \mathrm{L})$ & 1541 & $(527)$ \\
\hline
\end{tabular}

\footnotetext{
Values are shown as the mean (SD).

Abbreviations: BMI: body mass index, FFMI: fat-free mass index, FMI: fat mass index, FEV1: forced expiratory volume in one second, VC: vital capacity, HRCT: high resolution computed tomography, LAA: low-attenuation area, 6MWT: 6-minute walking test, CRP: C-reactive protein
}

dict FFM in patients with COPD. Since it was associated with both FFM and FM and also with BMI (data not shown), \%LAA is thought to be a useful predictor for body composition of COPD patients in a comprehensive manner. Though \%LAA was associated with both FFMI and FMI, FEV1\% predicted was related to FFMI but not to FMI. These results are thought to suggest the possibility that airflow limitation due to emphysema affects FFM more than
FM, which remains to be confirmed, including the relationship between the distribution of emphysema and body composition.

Secondly, it has also been reported that normal aging is associated with a progressive decline in the lean body mass and function, a phenomenon known as sarcopenia due to aging per se in the majority of patients with COPD. Sarcopenia is seen in general biological aging (28), and it is re- 
Inter Med 48: 41-48, 2009 DOI: 10.2169/internalmedicine.48.1102

Table 2. Correlations between FFMI or FMI and Clinical Variables by Univariate Analyses

\begin{tabular}{|c|c|c|c|c|c|}
\hline \multirow{2}{*}{ Variable } & \multirow{2}{*}{$\mathrm{n}$} & \multicolumn{2}{|c|}{ FFMI } & \multicolumn{2}{|c|}{ FMI } \\
\hline & & Correlation & p-value & Correlation & $\mathrm{p}$-value \\
\hline Age (yrs) & 112 & -0.444 & $<0.0005$ & 0.078 & 0.415 \\
\hline Pack-years & 112 & 0.064 & 0.502 & 0.21 & 0.026 \\
\hline Total protein $(\mathrm{g} / \mathrm{dL})$ & 112 & 0.012 & 0.900 & 0.067 & 0.484 \\
\hline $\begin{array}{l}\text { Total number of } \\
\text { lymphocytes }(/ \mu \mathrm{L})\end{array}$ & 112 & 0.229 & 0.015 & 0.125 & 0.189 \\
\hline Handgrip strength (kg) & 112 & 0.619 & $<0.0005$ & 0.055 & 0.567 \\
\hline 6MWT distance (m) & 112 & 0.376 & $<0.0005$ & -0.054 & 0.570 \\
\hline FEV1 \% predicted $(\%)$ & 112 & 0.447 & $<0.0005$ & 0.075 & 0.433 \\
\hline$\%$ LAA $(\%)$ & 112 & -0.459 & $<0.0005$ & -0.329 & $<0.0005$ \\
\hline $\mathrm{DLCO}(\mathrm{mL} / \mathrm{m} / \mathrm{mmHg})$ & 112 & 0.502 & $<0.0005$ & 0.115 & 0.228 \\
\hline $\begin{array}{l}\text { Highly-sensitive CRP } \\
(\mathrm{mg} / \mathrm{dL})\end{array}$ & 75 & -0.36 & 0.002 & -0.05 & 0.671 \\
\hline Adiponectin $(\mu \mathrm{g} / \mathrm{mL})$ & 34 & -0.329 & 0.058 & -0.391 & 0.022 \\
\hline
\end{tabular}

Correlations were shown as Pearson's bivariate linear correlation coefficient.

See Table 1 for abbreviations.

Table 3. Results of Multivariate Linear Regression Analysis of FFMI

\begin{tabular}{lccccc}
\hline \multirow{2}{*}{ Dependent variables } & \multicolumn{5}{c}{ Regression coefficient } \\
\cline { 2 - 6 } & Value & SEM & $95 \%$ CI & Standardized & \\
\hline Handgrip strength $(\mathrm{kg})$ & 0.135 & 0.016 & $0.104-0.166$ & 0.619 & $<0.0005$ \\
\%LAA $(\%)$ & -0.047 & 0.008 & $-0.062--0.031$ & -0.386 & $<0.0005$ \\
\hline
\end{tabular}

1. Predictive parameter was FFMI, and clinical parameters with significant correlations on univariate analyses except for FEV1\% predicted and DLCO (age, total number of lymphocytes, handgrip strength, distance on 6 MWT, and \%LAA as shown in Table 2) were used as dependent variables for stepwise analysis.

2. Dependent variables with p-values of less than 0.05 are shown.

See Table 1 for abbreviations.

ported that skeletal muscle strength could be a predictor of all-cause mortality in healthy men (29). Calorie restriction affects sarcopenia and limits the regeneration of muscle fibers, and recent data indicate that mitochondrial DNA deletion mutations and associated electron transport system enzymatic abnormalities are implicated in the loss of skeletal muscle fibers with age (12). FM also accumulates in the aging process, and it is considered to be important to reduce FM to decrease the impact of diabetes, vascular disease, and other complications of obesity (30). It was shown in the present study that FFMI in COPD was significantly and inversely correlated with aging per se, but not with FMI. 

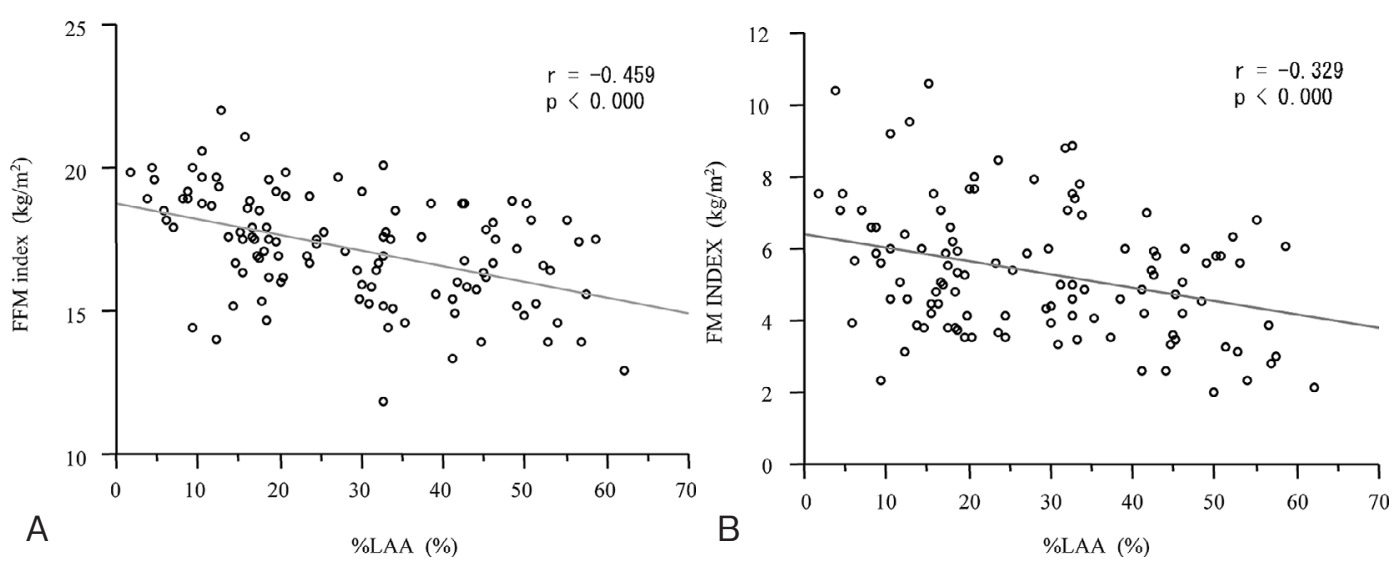

Figure 1. Scatter plots of the FFMI and FMI against \% LAA in 112 male COPD patients (Panels $A$ and B, respectively). Regression lines are also shown. See Table 1 for abbreviations.

Table 4. Results of Multivariate Linear Regression Analysis of FMI

\begin{tabular}{lccccc}
\hline \multirow{2}{*}{ Dependent variables } & \multicolumn{5}{c}{ Regression coefficient } \\
\cline { 2 - 5 } & Value & SEM & $95 \%$ CI & Standardized & p-value \\
\hline \%LAA (\%) & -0.042 & 0.010 & $-0.062--0.022$ & -0.361 & 0.022 \\
Pack-years & 0.012 & 0.004 & $0.004-0.019$ & 0.255 & 0.019 \\
\hline
\end{tabular}

1. Predictive parameter was FMI, and clinical parameters with significant correlations on univariate analyses (\%LAA or pack-years as shown in Table 2) were used as dependent variables for stepwise analysis.

2. Dependent variables with p-values of less than 0.05 are shown.

See Table 1 for abbreviations.

However, after multivariate analysis, it was found that age was not an independent determinant for FFMI. There are many causes of muscle-wasting in patients with COPD who show muscle weakness and atrophy, particularly in the extremities. These patients experience frequent exacerbations, which in turn results in malaise, anorexia, and inactivity due to such muscle weakness and atrophy, leading to a vicious cycle (31). Breathing effort and a high metabolic rate also contribute to weight loss in advanced COPD (32). Furthermore, systemic corticosteroid therapy promotes an increase in FM and a reduction in $\operatorname{FFM}(33,34)$. All of these factors may be inter-related and contribute to muscle wasting.

Third, the present data identified a correlation between FFMI and biomarkers of inflammatory changes, namely, highly-sensitive CRP ( $\mathrm{r}=-0.36, \mathrm{p}<0.002)$, although this correlation was excluded after the multivariate analysis. It is known that there are many similar biomarkers, including IL6 , IL-8, and TNF-alpha, which play a major role in promoting lipolysis and inhibiting insulin signaling, thereby inducing insulin resistance and changing FFM or FM (35).

Fourth, it is of interest to note that the data of both packyears and adiponectin were also associated with FM in
COPD (Table 2). It has been reported that smoking is related to the body FM in a general population sample (36), which is consistent with the present results. The role of adiponectin has been studied in various lung diseases, including bronchial asthma (37); however, to the best of our knowledge, only a few previous reports have investigated the role of adiponectin in COPD (38). Shore suggested that adiponectin has an anti-inflammatory effect in asthma (37); if this is also true in COPD, there is a possibility that adiponectin has a protective effect against chronic inflammation in COPD in cases of a low FM, which is consistent with a recent report (38). FM alone is reported as an independent risk factor of myocardial infarction (18), which is also known as one of the comorbidities of COPD (1). Thus, we raise a hypothesis that a low FM has a pivotal role connected with increased serum adiponectin, and subsequently suppresses chronic inflammation in COPD. However, it is necessary to be careful regarding this speculation, since it has recently been suggested that the normal range of BMI and cutoff points for obesity should be lower in Asians compared to Caucasians (39). It has been speculated that FM could have a marked effect on comorbidities, particu- 
larly for cardiovascular risk in COPD subjects; however, Western BMI criteria should not be applied to Asians, whose BMI is much lower (39).

In the stage I or II in COPD, the accumulation of fat mass partly due to smoking could be a risk for cardiovascular diseases. Recently, several reports support that the coexistence of obesity or metabolic syndrome occurs in the stage I or II in COPD $(40,41)$. However, the more emphysema (and/or COPD) progresses, the less fat mass, and the risk of cardiovascular disease could be lower in this aspect. In severe emphysema, possibly, the increment of adiponectin could also have a protective effect against chronic inflammation, which also could lead to the protection against cardiovascular diseases. Clearly, it is necessary to investigate the role of FM in COPD, and also to examine the ethnic differences in terms of the cardiovascular risk with a correlation between adiponectin and FM or body mass index in COPD.

Several limitations in the study design should be discussed, although we have identified interesting interactions between the severity of emphysema on HRCT in patients with COPD, and both FFM and FM. First, our population included smokers without COPD or another word, "stage 0" COPD subjects according to ATS/ERS guidelines (23); however, stage 0 has been deleted from the current GOLD updated guidelines (1). It is still controversial whether patients with stage 0 , because of being without airflow limitation, should be considered in the diagnosis of COPD as only at risk or showing the true type of COPD (1). In this study, when we excluded stage 0 subjects, \%LAA correlated with FM significantly, but FFM did not $(\mathrm{p}=0.09)$. Clearly, further investigation is needed to confirm the present results in a larger population including or excluding stage 0. Second, FFM and FM were measured by bioelectrical impedance analysis in this study. Data obtained by MRI or DEXA could be superior, although bioelectrical impedance analysis is an established and practical method to assess FFM or FM (42). Finally, the results of highly-sensitive CRP and adiponectin came from a small sample in this study, and so further studies with larger populations are needed.

In conclusion, the extent of emphysema was correlated with both FFM and FM in COPD. The more emphysema progresses, the more both FFM and FM would be reduced, which is thought to not only lead to increment of COPD mortality but also make the status of COPD more complicated, especially in the aspect of comorbidities. Because exercises could change body composition, evaluation of \% LAA could be critical to select the subjects for rehabilitation in COPD patients. Thus, evaluation of emphysema by HRCT may possibly serve not only as a prognostic marker but also as a marker to select targets for the treatment of COPD including pulmonary rehabilitation.

\section{Acknowledgement}

This study was funded by the Environmental Restoration and Conservation Agency in Japan.

\section{References}

1. Global Strategy for the Diagnosis, Management and Prevention of COPD, Global Initiative for Chronic Obstructive Lung Disease (GOLD), 2006. www.goldcopd.org/. Date last updated: December 2006. Date last accessed: July 30, 2007.

2. Bernard S, LeBlanc P, Whittom F, et al. Peripheral muscle weakness in patients with chronic obstructive pulmonary disease. Am J Respir Crit Care Med 158: 629-634, 1998.

3. Engelen MP, Schols AM, Does JD, Wouters EF. Skeletal muscle weakness is associated with wasting of extremity fat-free mass but not with airflow obstruction in patients with chronic obstructive pulmonary disease. Am J Clin Nutr 71: 733-738, 2000.

4. Pitta F, Troosters T, Spruit MA, et al. Characteristics of physical activities in daily life in chronic obstructive pulmonary disease. Am J Respir Crit Care Med 171: 972-977, 2005.

5. Debigare R, Marquis $\mathrm{K}$, Cote $\mathrm{CH}$, et al. Catabolic/anabolic balance and muscle wasting in patients with COPD. Chest 124: 8389, 2003.

6. Gosker HR, Kubat B, Schaart G, et al. Myopathological features in skeletal muscle of patients with chronic obstructive pulmonary disease. Eur Respir J 22: 280-285, 2003.

7. Gosker HR, Engelen MP, van Mameren $\mathrm{H}$, et al. Muscle fiber type IIX atrophy is involved in the loss of fat-free mass in chronic obstructive pulmonary disease. Am J Clin Nutr 76: 113-119, 2002.

8. Agusti AG, Sauleda J, Miralles C, et al. Skeletal muscle apoptosis and weight loss in chronic obstructive pulmonary disease. Am J Respir Crit Care Med 166: 485-489, 2002.

9. Kaizu Y, Ohkawa S, Odamaki M, et al. Association between inflammatory mediators and muscle mass in long-term hemodialysis patients. Am J Kidney Dis 42: 295-302, 2003.
10. Filippatos GS, Kanatselos C, Manolatos DD, et al. Studies on apoptosis and fibrosis in skeletal musculature: a comparison of heart failure patients with and without cardiac cachexia. Int J Cardiol 90: 107-113, 2003.

11. Visser M, Kritchevsky SB, Newman AB, et al. Lower serum albumin concentration and change in muscle mass: the Health, Aging and Body Composition Study. Am J Clin Nutr 82: 531-537, 2005.

12. Bua E, McKiernan SH, Aiken JM. Calorie restriction limits the generation but not the progression of mitochondrial abnormalities in aging skeletal muscle. Faseb J 18: 582-584, 2004.

13. Husom AD, Ferrington DA, Thompson LV. Age-related differences in the adaptive potential of type I skeletal muscle fibers. Exp Gerontol 40: 227-235, 2005.

14. Bar-Shai M, Carmeli E, Coleman R, Reznick AZ. Mechanisms in muscle atrophy in immobilization and aging. Ann N Y Acad Sci 1019: 475-478, 2004.

15. Gillette Guyonnet $\mathrm{S}$, Abellan Van Kan G, Alix E, et al. IANA (International Academy on Nutrition and Aging) Expert Group: weight loss and Alzheimer's disease. J Nutr Health Aging 11: 3848, 2007.

16. Schols AM, Slangen J, Volovics L, Wouters EF. Weight loss is a reversible factor in the prognosis of chronic obstructive pulmonary disease. Am J Respir Crit Care Med 157: 1791-1797, 1998.

17. Agusti AG, Noguera A, Sauleda J, et al. Systemic effects of chronic obstructive pulmonary disease. Eur Respir J 21: 347-360, 2003.

18. Nicklas BJ, Penninx BW, Cesari M, et al. Association of visceral adipose tissue with incident myocardial infarction in older men and women: the Health, Aging and Body Composition Study. Am 
J Epidemiol 160: 741-749, 2004.

19. Broekhuizen R, Wouters EF, Creutzberg EC, Schols AM. Raised CRP levels mark metabolic and functional impairment in advanced COPD. Thorax 61: 17-22, 2006.

20. Nakano Y, Muro S, Sakai H, et al. Computed tomographic measurements of airway dimensions and emphysema in smokers. Correlation with lung function. Am J Respir Crit Care Med 162: 1102-1108, 2000

21. Oh DK, Ciaraldi T, Henry RR. Adiponectin in health and disease. Diabetes Obes Metab 9: 282-289, 2007.

22. Fukuchi $Y$, Nishimura M, Ichinose M, et al. COPD in Japan: the Nippon COPD Epidemiology study. Respirology 9: 458-465, 2004.

23. American Thoracic Society / European Respiratory Society Task Force. Standards for the Diagnosis and Management of Patients with COPD [Internet]. Version 1.2. New York: American Thoracic Society; 2004 http://www.thoracic.org/go/copd. Date last updated: September 8 2005. Date last accessed: July 302007.

24. Pinto-Plata VM, Mullerova H, Toso JF, et al. C-reactive protein in patients with COPD, control smokers and non-smokers. Thorax 61: 23-28, 2006.

25. ATS statement: guidelines for the six-minute walk test. Am J Respir Crit Care Med 166: 111-117, 2002.

26. Standardization of Spirometry, 1994 Update. American Thoracic Society. Am J Respir Crit Care Med 152: 1107-1136, 1995.

27. Japanese Respiratory Society. The predicted values of spirometry and arterial blood gas analysis in Japanese. J Jpn Resp Soc 39: Appendix, 2001 (in Japanese).

28. Doherty TJ. Invited review: Aging and sarcopenia. J Appl Physiol 95: 1717-1727, 2003

29. Metter EJ, Talbot LA, Schrager M, Conwit R. Skeletal muscle strength as a predictor of all-cause mortality in healthy men. J Gerontol A Biol Sci Med Sci 57: B359-B365, 2002.

30. Kennedy RL, Chokkalingham K, Srinivasan R. Obesity in the elderly: who should we be treating, and why, and how? Curr Opin Clin Nutr Metab Care 7: 3-9, 2004.
31. Koehler F, Doehner W, Hoernig S, et al. Anorexia in chronic obstructive pulmonary disease--association to cachexia and hormonal derangement. Int J Cardiol 119: 83-89, 2007.

32. Mannix ET, Manfredi F, Farber MO. Elevated O2 cost of ventilation contributes to tissue wasting in COPD. Chest 115: 708-713, 1999.

33. Pijl H, Meinders AE. Bodyweight change as an adverse effect of drug treatment. Mechanisms and management. Drug Saf 14: 329342, 1996.

34. Calverley PM. The role of corticosteroids in chronic obstructive pulmonary disease. Semin Respir Crit Care Med 26: 235-245, 2005.

35. Warne JP. Tumour necrosis factor alpha: a key regulator of adipose tissue mass. J Endocrinol 177: 351-355, 2003.

36. Bamia C, Trichopoulou A, Lenas D, Trichopoulos D. Tobacco smoking in relation to body fat mass and distribution in a general population sample. Int J Obes Relat Metab Disord 28: 1091-1096, 2004.

37. Shore SA. Obesity and asthma: implications for treatment. Curr Opin Pulm Med 13: 56-62, 2007.

38. Tomoda K, Yoshikawa M, Itoh $\mathrm{T}$, et al. Elevated Circulating Plasma Adiponectin in Underweight Patients With COPD. Chest 132: 135-140, 2007.

39. Razak F, Anand SS, Shannon H, et al. Defining obesity cut points in a multiethnic population. Circulation 115: 2111-2118, 2007.

40. Steuten LM, Creutzberg EC, Vrijhoef HJ, Wouters EF. COPD as a multicomponent disease: inventory of dyspnoea, underweight, obesity and fat free mass depletion in primary care. Prim Care Respir J 15: 84-91, 2006.

41. Marquis K, Maltais F, Duguay V, et al. The metabolic syndrome in patients with chronic obstructive pulmonary disease. J Cardiopulm Rehabil 25: 226-232, 2005.

42. Kyle UG, Bosaeus I, De Lorenzo AD, et al. Bioelectrical impedance analysis--part I: review of principles and methods. Clin Nutr 23: 1226-1243, 2004.

(C) 2009 The Japanese Society of Internal Medicine http://www.naika.or.jp/imindex.html 